\title{
A RARE CASE OF METASTATIC MELANOMA IN THE BREAST
}

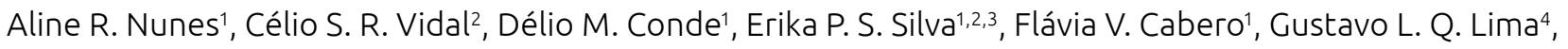
Kauana C. S. Nascimento4, Rogério B. Ferreira ${ }^{1,2}$

${ }^{1}$ Mastology Service, Hospital Materno Infantil de Goiânia - Goiânia (GO), Brazil.

${ }^{2}$ Mastology Service, Hospital Geral de Goiânia - Goiânia (GO), Brazil.

${ }^{3}$ Mastology Department, Hospital Geral de Goiânia - Goiânia (GO), Brazil.

${ }^{4}$ Hospital Geral de Goiânia - Goiânia (GO), Brazil.

Objective: To report a case of metastatic melanoma in the breast. Case: woman, 66 years, complaining of mass in the left breast. She reported treatment of melanoma in the gluteal region in 2011 and left breast cancer in 2016 (luminal B (RH+, HER2-), undergoing mastectomy with sentinel lymph node biopsy, chemotherapy and hormone therapy). Physical examination: breast implants; regular, mobile mass, $10 \mathrm{~h}$ of left breast, measuring about $2.0 \mathrm{~cm}$. Ultrasonography showed a solid-cystic mass, predominantly solid, with a hypoechogenic content, with its largest axis parallel to the skin, with slightly lobed contours and well-defined limits, with an apparent increase in the surrounding echogenicity, at 10 hours of the left breast, measuring 1.9 x 1.7 × $0.9 \mathrm{~cm}$, suggestive of complex cyst, BI-RADS 4. Mammography: mass partially visualized in the medial quadrants of the left breast, visible, only visible in the craniocaudal view. The hypothesis was that of recurrence of breast cancer. Patient underwent extensive excision of the lesion to obtain surgical margins of neoplasia. Histopathological findings, associated with immunohistochemistry (AE1/AE3: negative. S-100 protein, melan A and Hmb45: positive), were compatible with the diagnosis of metastatic melanoma for the breast. Subsequently, a recurrence of melanoma in the gluteus was diagnosed. Chest CT demonstrated multiple lung lesions. Patient is being treated for melanoma, and using letrozole. Conclusion: metastatic melanoma for the breast is uncommon. The breast surgeon should be aware of this possibility, especially in women with a history of melanoma. In addition, there is a need to work together with the pathologist, making diagnosis and treatment as soon as possible. 